\title{
Genèse du récit maghrébin pour enfants. Le cas de Mohammed Dib
}

Guy Dugas

\section{(2) OpenEdition}

1 Journals

Édition électronique

URL : http://journals.openedition.org/genesis/4061

DOI : 10.4000/genesis.4061

ISSN : 2268-1590

Éditeur :

Presses universitaires de Paris Sorbonne (PUPS), Société internationale de génétique artistique littéraire et scientifique (SIGALES)

Édition imprimée

Date de publication : 1 juin 2019

Pagination : 81-92

ISSN : 1167-5101

\section{Référence électronique}

Guy Dugas, «Genèse du récit maghrébin pour enfants. Le cas de Mohammed Dib », Genesis [En ligne], 48 | 2019, mis en ligne le 01 juin 2020, consulté le 24 janvier 2021. URL : http://

journals.openedition.org/genesis/4061 ; DOI : https://doi.org/10.4000/genesis.4061 


\section{Genèse du récit maghrébin pour enfants. Le cas de Mohammed Dib}

Guy Dugas

$\mathbf{J}$ e pourrais, pour commencer, présenter un large inventaire de la production de récits maghrébins pour enfants : il surprendrait sans doute par sa richesse, aussi bien en langue française qu'en langue arabe. Ou proposer une biobibliographie de Mohammed Dib - dont les œuvres comptent parmi les plus importantes de la littérature maghrébine de langue française - afin de montrer comment sa production pour les enfants prend place au sein de cet ensemble : ainsi j'ai été surpris de noter que cette production n'apparaît pas dans de nombreuses bibliographies le concernant; qu'elle a été fort peu étudiée et qu'il est très difficile de trouver des éléments d'analyse.

Pour plus d'efficacité, j'ai préféré limiter cette approche aux quatre récits que l'écrivain algérien Mohammed Dib (1920-2003) a produits pour la jeunesse. Je les indexe ici par ordre chronologique de publication :

- Baba Fekrane, Paris, La Farandole (ill. Mireille Miailhe), 1959;

- L'Histoire du chat qui boude (ill. Bernard Domenger), Paris, La Farandole, 1974. Rééd Messidor-La Farandole, 1980 (ill. Jean-Claude Luton) et Albin Michel Jeunesse, 2003 (ill. Christophe Merlin);

- L'Hippopotame qui se trouvait vilain (ill. Emmanuel Kerner), Paris, Albin Michel Jeunesse, 2001;

- Salem et le sorcier (ill. Alexis Logié), Rabat, Yomad, 2000 ; rééd. bilingue : Salem et le sorcier / Salim wa sahîr (ill. Virginie Soumagnac), Rabat, Yomad, 2003.

Afin de tenir compte de l'orientation de Genesis et du matériel dont je dispose, je privilégierai dans cet article l'analyse de deux de ces récits : L'Histoire du chat qui boude et L'Hippopotame qui se trouvait vilain, qui sont les deux œuvres pour lesquelles nous disposons d'un matériel génétique.

\section{Considérations générales, formelles et thématiques sur le corpus}

Abordons ce corpus par quelques remarques d'ensemble :

- Hormis Baba Fekrane, recueil de contes du terroir algérien, ces récits ont en commun de s'adresser à de tout jeunes enfants (5 à 7 ans), ce qui, sur le plan formel et narratologique, induit quelques conséquences : prééminence de l'illustration et simplicité tant lexicale que syntaxique du texte l'accompagnant. Ce sont donc des ouvrages de nature composite : avec du texte et de l'image (de façon générale, dans les récits pour enfants, plus le public visé est jeune, plus la part de l'illustration l'emporte sur le texte), et donc des ouvrages de collaboration plus ou moins étroite entre un auteur et un illustrateur.

- Si l'on excepte une fois encore certains textes de Baba Fekrane, ce sont à peu près tous des contes dits «de randonnée», ou «d'inventaire», ce qui ne manque pas d'avoir des conséquences sur la structure du récit, sur lesquelles nous reviendrons.

- Ils puisent assez largement dans la tradition orale maghrébine, dont ils gardent certaines marques. La formule : «Nous sommes allés tout le long du chemin / de la route et nous avons trouvé un sac de perles : les grosses pour moi, les petites pour toi...», que l'on retrouve en clausule de tous les contes de Dib1, m'a causé quelques soucis d'identification ${ }^{2}$, étant en effet absente, sous cette forme, dans la tradition arabe ou berbère où l'idée de marche est pourtant fort présente ${ }^{3}$.

- On note une énorme prééminence de la thématique animalière : grenouille, chat, âne, oiseaux, hérisson,

1. Sauf dans Salem et le sorcier, ce qui peut paraître paradoxal lorsqu'on sait que ce conte est purement et simplement extrait d'un roman de Dib où non seulement la formule figure, mais où, en plus, elle est l'objet d'un dialogue entre le conteur et son public (voir plus loin l'analyse du conte et de ses sources).

2. Selon William Marçais, il s'agirait d'un particularisme très localisé dans la région natale de Dib (Le Dialecte arabe parlé à Tlemcen, Paris, Leroux, 1902, p. 260).

3. Voir par exemple les Contes berbères de Kabylie recueillis par Jean et Marguerite-Taos Amrouche. 
hippopotame..., ce qui doit assez peu étonner, la littérature enfantine universelle puisant largement dans ce champ.

- Les deux premiers récits pour enfants de Mohammed Dib lui ont été commandés par les éditions La Farandole (devenues Messidor-La Farandole en 1979), fondées en 1955 par le Parti communiste français, où Aragon - dont Dib, un moment membre du PCA, puis «compagnon de route» du PCF, a été proche des années cinquante aux années soixante-dix ${ }^{4}$ - joue un rôle important. Lorsque l'écrivain passe chez Albin Michel, Michel Piquemal, directeur de la collection « Jeunesse», lui commande L'Hippopotame qui se trouvait vilain, puis une nouvelle version de L'Histoire $d u$ chat qui boude qui ne paraîtra qu'à titre posthume.

- Le récit pour enfants de Mohammed Dib le plus récent a été publié au Maroc, en version bilingue français/arabe, dans une maison d'édition, Yomad, spécialisée en littérature jeunesse, ce qu'il est intéressant de noter, il me semble, quant à l'économie du livre pour enfants au Maghreb.

- Il est difficile d'avoir des indications sur la relation entre l'auteur et l'illustrateur. Si les deux premiers ouvrages ont été illustrés par des dessinateurs militants, rattachés à la maison d'édition 5 , le cas du dernier livre est intéressant, nous en reparlerons.

- Enfin (et cela en dit long sur la considération qu'accordent les Belles Lettres à ce corpus paralittéraire), l'archive des récits pour enfants a souvent été négligée, peu ou mal constituée, la plupart du temps par les auteurs euxmêmes - et plus encore chez les écrivains francophones! Si bien qu'en dehors du dossier très intéressant de L'Histoire du chat qui boude, il reste peu de chose des avant-textes des récits de Mohammed Dib, dont les archives, d'un volume impressionnant, ont été déposées à la $\mathrm{BnF}^{6}$.

Cependant, grâce aux brouillons de L'Histoire du chat qui boude et de L'Hippopotame qui se trouvait vilain conservés dans le fonds Dib de la BnF, nous allons pouvoir étudier la genèse auctoriale puis éditoriale de deux récits pour la jeunesse, de manière très développée pour l'un, parce que les brouillons manuscrits présentent un grand intérêt, beaucoup moins pour l'autre, parce que nous ne disposons que d'une dactylographie très propre.

Nous y ajouterons ensuite quelques réflexions complémentaires sur les deux autres récits, le plus ancien : Baba Fekrane (1959) et le plus récent : Salem et le sorcier (2003), dont nous n'avons pu retrouver les avant-textes.

\section{L'Histoire du chat qui boude}

\section{Genèse éditoriale}

L'Histoire du chat qui boude a une genèse éditoriale assez exceptionnelle : publiée initialement en 1974 (format à l'italienne $21,5 \times 20$; couverture brochée; illustrations de Bernard Domenger; réimpression à l'identique deux ans plus tard), elle est rééditée deux fois, en 1980 et 2003, chez des éditeurs et avec des illustrateurs différents.

En 1980, les éditions La Farandole rééditent le récit sous couverture reliée, dans un format presque carré $(22,5 \times 24,5)$ et avec des illustrations de Jean-Claude Luton, peintre et illustrateur très connu. Une lecture comparative des deux textes de 1974 et 1980 montre qu'ils sont exactement semblables, seule l'illustration variant.

En 2003, l'ultime version, publiée chez Albin Michel Jeunesse, présente un texte assez différent des précédents, sous une belle couverture reliée et dans un grand format $(26,5 \times 34,5)$, qui omet de faire référence, dans les mentions légales, à l'édition originale 7 . Très colorées, les illustrations pleines pages sont de Christophe Merlin ${ }^{8}$, peintre et auteur de bandes dessinées.

Voilà qui permet à la fois un travail sur les différentes iconographies et, à partir du remarquable dossier de brouillons, une approche véritablement génétique.

\section{Comparaison des trois versions publiées}

À l'origine, l'histoire, assez similaire d'une version à l'autre, présente trois personnages principaux. Vieux-Père

4. Sur la relation Dib-Aragon, voir notre contribution «Dib-Aragon, l'amitié au risque de l'engagement », dans l'ouvrage collectif Mohammed Dib écrivain de lumière publié en 2017 par les éditions Sédia à Alger, sous la direction de Yahia Belaskri, p. 123-135.

5. C'est notamment le cas de l'illustratrice de Baba Fekrane, Mireille Miailhe, née Glodek (1921-2010), militante du PCF, engagée contre la guerre d'Algérie et proche d'Aragon.

6. L'archive des récits pour enfants de Mohammed Dib est conservée dans la boîte 38bis de cet ensemble cote Naf 28679 (69). Je remercie Colette Dib d'avoir permis sa consultation et la reproduction de quelques extraits des manuscrits.

7. Le copyright porte seulement la mention : «Une première version de cette histoire, illustrée par Jean-Claude Luton, [...] éditée chez Scanéditions - La Farandole en 1980. »

8. Christophe Merlin est devenu depuis l'un des illustrateurs jeunesse français les plus connus. 


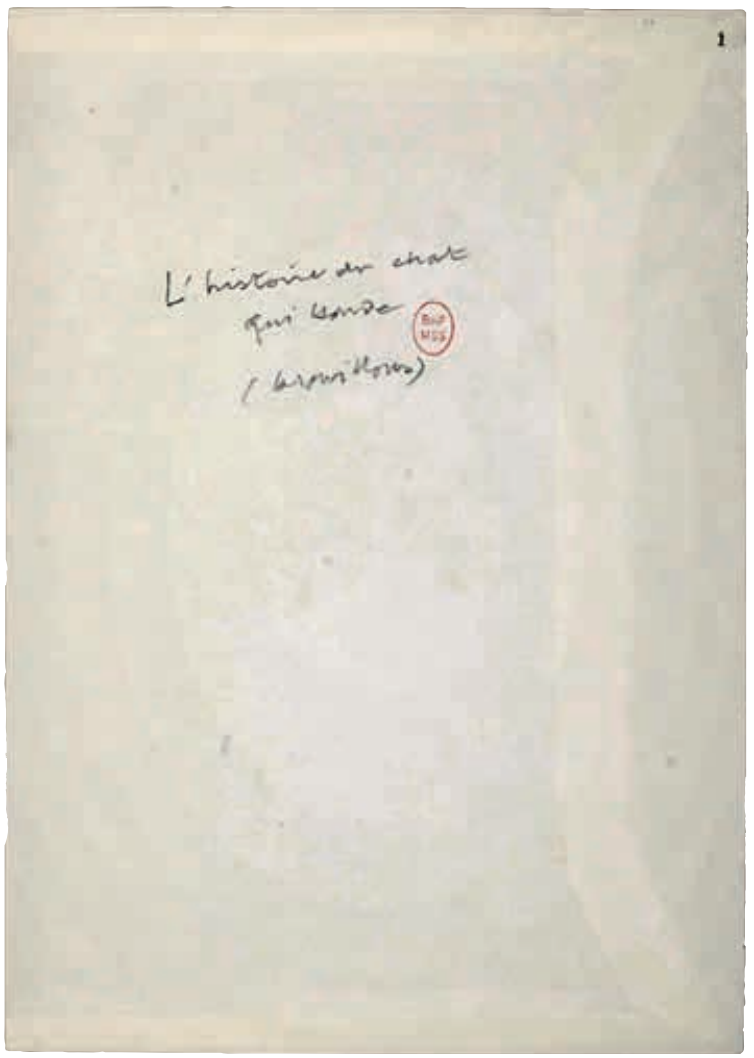

Fig. 1 : Page de titre de L'Histoire du chat qui boude. Boîte 38bis, cote Naf 28679, BnF.

Avec l'aimable autorisation de la famille Dib. (C) Bibliothèque nationale de France

a ramené des grives de la chasse, Vieille-Mère est chargée de les faire rôtir. Face à eux, le chat de la maison, injustement accusé par Vieille-Mère de les avoir mangées ${ }^{9}$, s'en va bouder sur la terrasse, ce qui conduit à une chaîne de solidarité naturelle : la terrasse s'écroule; un mur perd son crépi ; un oiseau s'arrache les plumes ; l'arbre sur lequel il est perché se dépouille de ses feuilles; à côté, une source ne tarde pas à se tarir; au grand dam de la servante qui brise sa cruche; la maîtresse, dépitée de ne pas avoir d'eau, déchire sa robe; son mari, voyant sa robe déchirée, la bat; le boulanger, enfin, qui n'a pourtant fait que voir passer ce mari fâché, décide de se précipiter dans son four!

Si le déroulement de la diégèse reste globalement le même dans les trois versions - suivant le principe : petite cause $>$ grands effets, que Dib utilise dans nombre de ses récits pour enfants - la troisième version apporte de substantielles modifications qui changent totalement l'esprit et le rythme du texte.

En effet, les situations initiale et finale y sont nettement plus développées, dans un esprit totalement différent des deux précédentes versions : l'incipit s'ouvre sur une brève description du chat, inexistante dans les deux premières versions. Il s'agit de quelques lignes qui précisent le titre de l'ouvrage, caractérisent ce personnage majeur 10 et suscitent l'attente du lecteur par une double interrogation : «Il boude. Pourquoi ? Que s'est-il passé ?»-des effets typographiques (caractères gras de ces questions et corps augmenté qui sont des procédés d'accélération que l'on retrouvera tout au long du récit) et l'illustration concourant également à cette attente.

L'excipit a été lui aussi profondément modifié dans la troisième version où les personnages du mari et du boulanger ne font plus qu'un, ce que permet aisément la structure d'enchaînement du conte de randonnée. Ceci a pour double effet bénéfique :

- de faire l'économie de la séquence machiste du mari battant sa femme. Que diable ! d'une version à l'autre, nous sommes passés au $\mathrm{XXI}^{\mathrm{e}}$ siècle, et ce type de scène est par trop associé aux lieux communs que l'Occident nourrit sur la culture arabo-musulmane;

- de dédramatiser la fin du récit. Certes, le boulanger se précipite dans son four 11 comme dans les versions précédentes, mais cela n'en constitue plus le dénouement; la troisième version comporte deux doubles pages supplémentaires qui ramènent logiquement le lecteur au couple initial Vieux-Père - Vieille-Mère, et le récit se clôt sur un tête-à-tête assez comique en fin de compte, Vieux-Père promettant de ne plus jamais rapporter de grives en cadeau, et Vieille-Mère marmonnant «dans les poils de son menton» qu'elle n'en est que plus heureuse d'avoir mangé celles qu'elle devait faire rôtir!

9. Les thèmes des démêlés de la vieille et du vieux, ou des mensonges et roueries de la femme voleuse, mangeant la pitance rapportée par le mari puis accusant n'importe qui, voire n'importe quoi (et jusqu'à la marmite!) de l'avoir fait, sont deux classiques du conte populaire, arabe autant que berbère (voir Delphin, Recueil de textes pour servir à l'étude de l'arabe parlé, Paris-Alger, 1891, p. 46-48 et René Basset, Essai sur la littérature des Berbères, Alger, typographie J. Carbonel, 1920, p. 157-159).

10. Dans les deux premières versions, ce personnage n'est jamais caractérisé autrement que par sa bouderie, alors que cette séquence décrit un «grand chat, d'un beau noir [...] ventre allongé sur la terrasse dans la fière posture du Sphinx».

11. Avec une nuance sémantique de taille: dans les versions 1 et 2 , le boulanger dit: «et moi je me grillerai! » en se jetant dans son four, ce qui est modifié dans la version 3 en «Moi, par le Diable, je me jetterai dans mon four !» (en caractères gras et corps augmenté). 

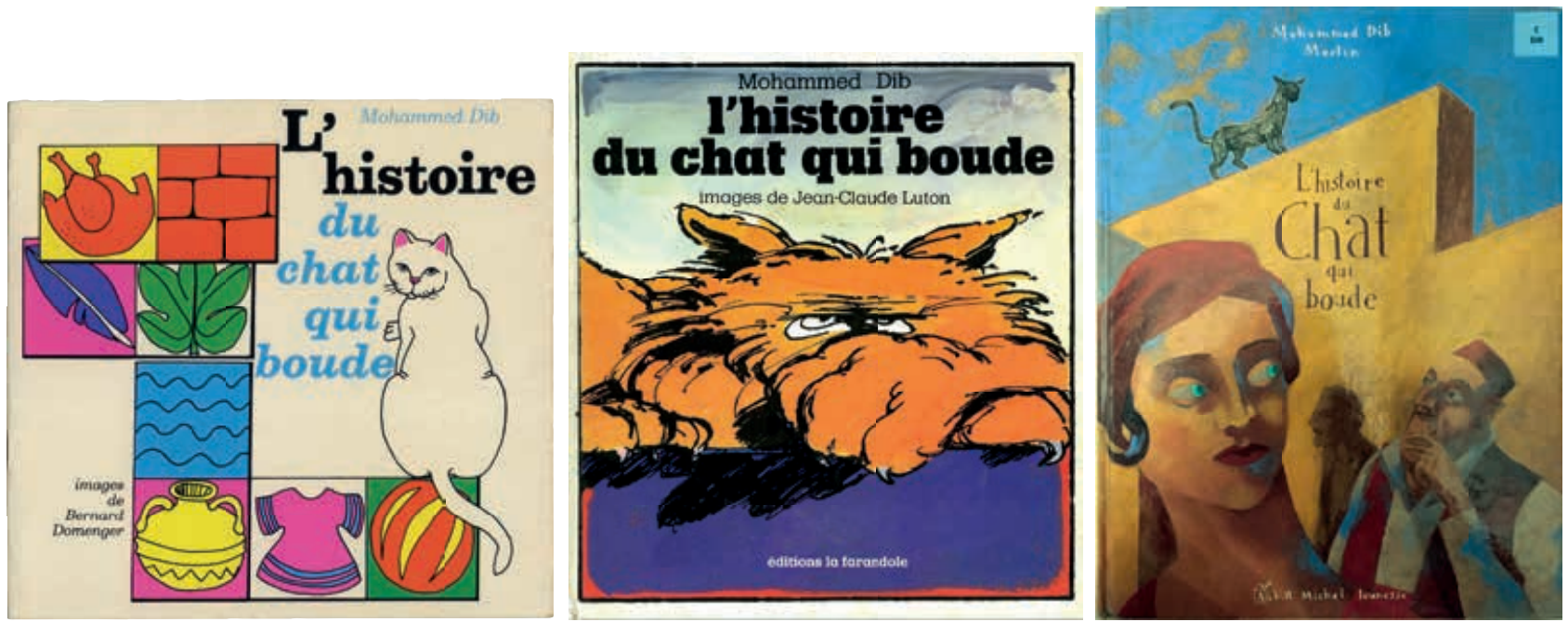

Fig. 2 : Trois couvertures de L'Histoire du chat qui boude :

a) celle de l'édition $1974(21,5 \times 20 \mathrm{~cm})$, b) celle de $1980(22,5 \times 24,5 \mathrm{~cm})$ et c) celle de $2003(26,50 \times 34,50 \mathrm{~cm})$.

Collection personnelle de l'auteur.

\section{Illustration et couvertures : comparaison des trois versions}

L'analyse comparative des trois couvertures et de l'illustration, venant en complément de l'étude des textes, est elle aussi révélatrice.

- Les couvertures (fig. 2 a, b, c)

La première chose qui saute aux yeux, avant même d'ouvrir les livres, c'est le symbolisme des trois couvertures, sur lesquelles le chat est évidemment présent, quoique sur des modes différents :

- un gros chat blanc trop peu boudeur sur la couverture de la première version dont l'illustration en damier suggère les différents rebondissements du récit;

- un gros matou couleur fauve sortant les griffes dans la deuxième version;

- dans la troisième version, au second plan, perché sur sa terrasse, le chat surplombe le couple Vieux-Père - VieilleMère. C'est la seule couverture à présenter d'autres protagonistes que le chat, dans un environnement architectural et vestimentaire typiquement arabe. Nous reviendrons plus loin sur le mode très particulier d'association des noms de l'auteur et de l'illustrateur sur la couverture de cette version.

Blanche et muette dans les deux premières versions, la quatrième de couverture reprend dans la troisième version, sur un fond très coloré, les quelques lignes de description du chat boudeur, avec, en guise d'invite à la lecture, la double interrogation déjà mentionnée.

\section{- L'illustration}

Marqueur des périodes auxquelles ont été publiés les ouvrages, l'illustration est fort différente d'une version à l'autre.
La première version est illustrée de dessins au trait de Bernard Domenger, en quatre ou cinq couleurs de base, de facture naïve, à motifs stéréotypés (maisons chaulées à toits plats, flore et faune africaine, vêtements et coiffes orientaux...). L'illustration occupe les pages de manière inégale, laissant quelquefois de grands espaces blancs.

Pour la deuxième version, sur le même texte, JeanClaude Luton nous offre une illustration très différente, plus personnelle, en couleurs sur la page de droite, en noir et blanc sur celle de gauche, sans la moindre volonté d'exotisme ou d'ancrage dans un contexte culturel précis. Les dessins de la page de droite, accompagnant le texte de Dib, illustrent fidèlement les différentes étapes de son récit. Ceux de la page de gauche, dépourvue de tout texte, présentent Vieille-Mère devant une vingtaine de grives, d'abord squelettique puis de plus en plus grosse au fur et à mesure qu'elle les dévore. L'iconographie seule y raconte ainsi une tout autre histoire, éludée par la narration dibienne, celle de Vieille-Mère emportée par son péché de gourmandise.

La troisième version est la plus équilibrée : graphie et iconographie s'y combinent le plus heureusement, l'ensemble fonctionnant plus à la manière d'une bande dessinée que d'un livre illustré ${ }^{12}$. Au texte, profondément remanié, introduisant, comme autant de bulles, des dialogues, des interrogations et des effets typographiques, l'illustrateur apporte la modernité de sa création et un peu de sa personnalité de bédéiste ${ }^{13}$, à travers de grandes planches couvrant le plus souvent la double page, qui créent un univers extrêmement vivant, peuplé de nombreux

12. Sentiment renforcé par le procédé éditorial qui consiste à associer en caractères identiques sur la couverture le nom de l'auteur et celui de l'illustrateur, amputé pour la circonstance de son prénom - ce que Christophe Merlin ne fait que dans ses bandes dessinées. Voir par exemple Nürburgring 57 (Glénat, 2012) et Renault sans limites (Glénat, 2013). 
personnages dont certains n'ont rien à voir avec le récit, un monde explicitement maghrébin, cependant dépourvu de toute vision stéréotypée.

Dès la première double page, celle où Dib introduit une description du chat, Merlin présente dans un contexte d'harmonie tous les actants du récit - hormis Vieille-Mère qui n'apparaîtra que plus tard, à travers une double page entièrement consacrée à son forfait. À cette entrée en matière fait écho à la fin de l'ouvrage, après toute une série de planches très animées, où les protagonistes de l'histoire figurent parmi quantité de personnages, une autre double page résumant par l'image, dans un contexte de désolation, toutes les conséquences du mensonge de Vieille-Mère.

\section{Étude des brouillons}

Les brouillons de L'Histoire du chat qui boude conservés dans le fonds Dib de la BnF se composent de onze feuillets entièrement manuscrits, non datés, rédigés au recto seulement - avec de nombreuses reprises et ratures. Certains d'entre eux figurent sur un papier quadrillé jauni, type cahier d'écolier de format $17 \times 24$ (fig. 3). Nous formulons l'hypothèse qu'il s'agit des brouillons de la version originale du texte, celle de l'édition de 1980.

Plus nombreux sont les brouillons au dos d'enveloppes en papier blanc, que Dib avait l'habitude d'utiliser entières ou découpées à divers formats, et numérotées. Les tampons de la poste, parfois nettement lisibles ${ }^{14}$, laissent inférer une datation dans la décennie 2000 (fig. 4a et b). Cette observation conduit à considérer les feuillets en question comme un brouillon de l'ultime réédition. Brouillon d'ailleurs incomplet, puisqu'il s'arrête à la séquence de la robe déchirée par la servante - c'est-à-dire qu'il y manque tout ce qui est apporté au dénouement par l'édition 2003, à savoir la fusion en un seul des personnages du mari et du boulanger et le face à face entre Vieux-Père et Vieille-Mère.

À partir de ce constat, il est possible d'imaginer que cet ensemble manuscrit constitue une reprise, sur les feuillets quadrillés, des brouillons de l'édition 1980, complétée et corrigée sur les feuillets blancs en vue de l'ultime édition. Ce que permet de vérifier une simple comparaison des manuscrits avec les versions publiées : ainsi la longue partie de mise en place du récit, présente seulement dans l'édition

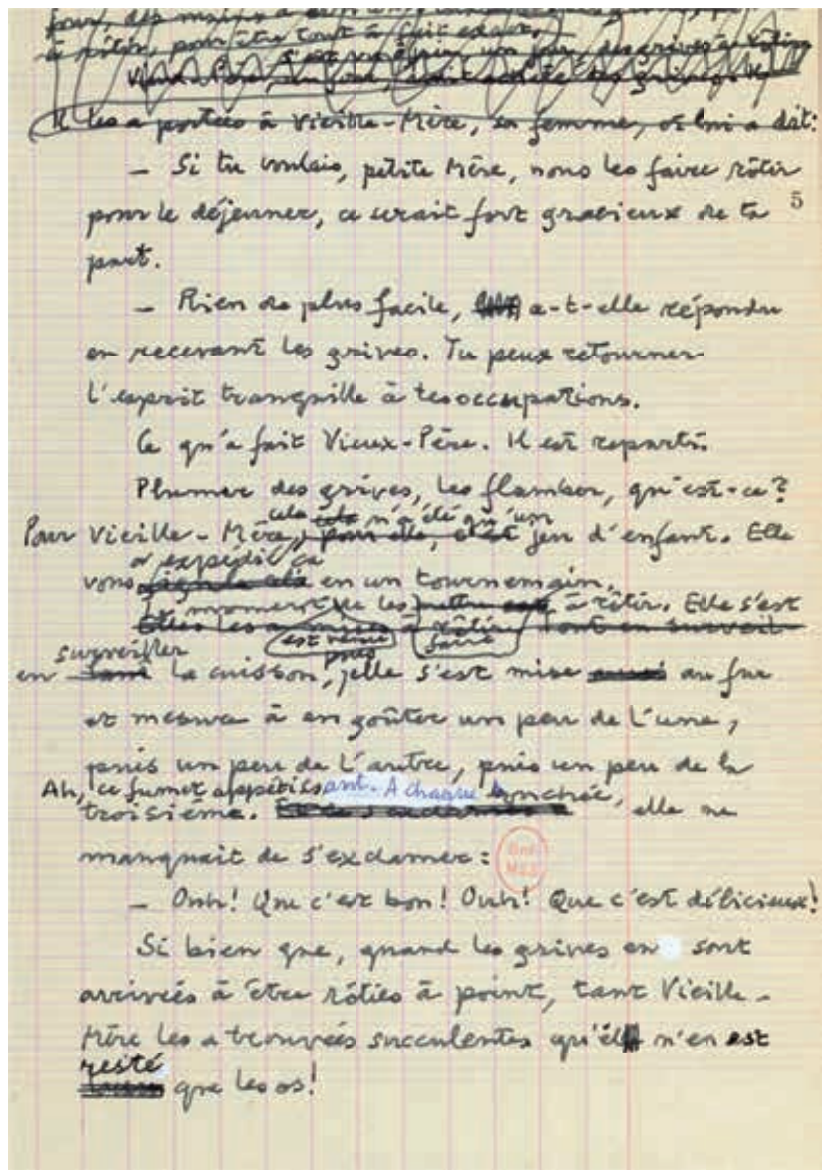

Fig. 3 : Boîte 38bis, cote Naf 28679.

Avec l'aimable autorisation de la famille Dib. (C) BnF

2003, apparaît abondamment travaillée sur l'un des feuillets blancs (fig. 5a), puis mise au net sur un autre feuillet blanc, lui-même contrecollé sur l'un des feuillets quadrillés portant le texte antérieur (fig. 5b).

Ajoutons, pour être complet, que quelques notes manuscrites sans le moindre rapport avec le récit figurent sur certains de ces feuillets 15 .

13. Voir par exemple la double page où le face à face entre la servante et sa maîtresse se double d'un second entre le chat et une tortue, animal auquel Merlin a consacré un album en 1999, ou encore ces trois planches où s'exprime son goût des véhicules anciens à travers un motif que le texte n'appelle nullement : un petit garçon au volant d'une voiture à pédales. 14. L'une de ces lettres est adressée à Dib par le journal El Watan, quotidien algérien francophone connu, fondé fin 1990. Le tampon postal est illisible, mais sur une autre (fig. 4a), on lit aisément «Odéon, Boulevard Saint-Germain, 09.03.02».

15. Ainsi, au feuillet 3, cette réflexion : «facticité galopante de l'existence à force de eonditionnement /décervellement/ télévisuel tyrannie décervelage, d'obsession des jeux et /de/ décervelages télévisuels, de la religion des loisirs et de l'amusement» et au feuillet suivant cette mention : «Action directe (France)» que l'on distingue sur la fig. 4a. 


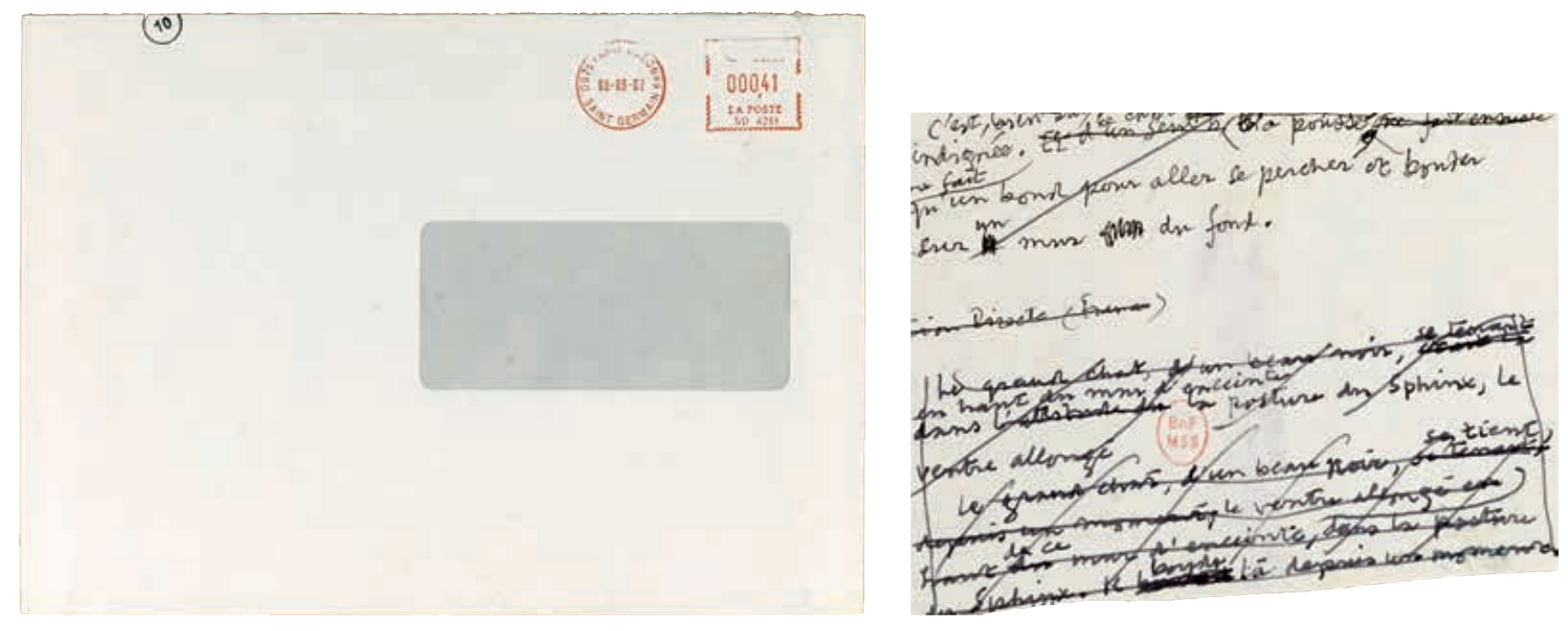

Fig. 4a et 4b. Recto et verso d'une enveloppe. L'Histoire du chat qui boude.

Avec l'aimable autorisation de la famille Dib. @) Bibliothèque nationale de France

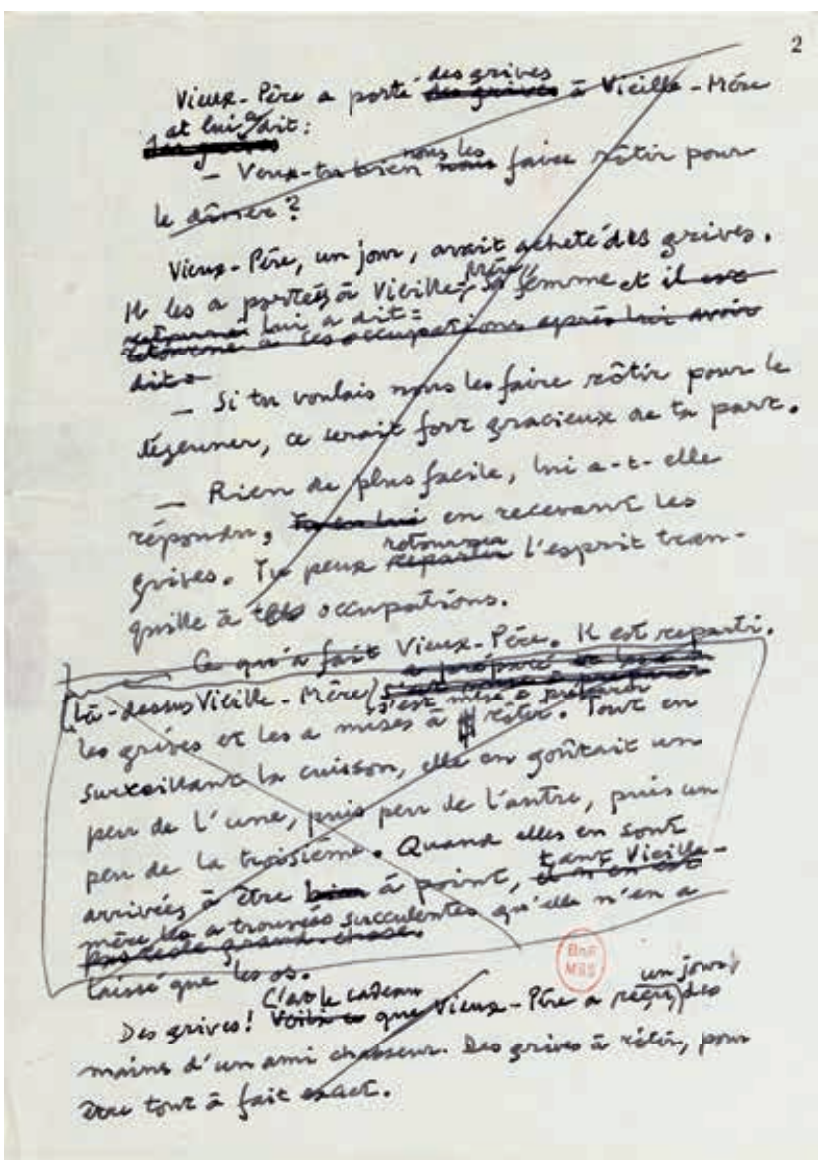

Fig. 5a : Travail sur incipit de la troisième version - Fol. 4 .

Boîte 38 bis, cote Naf 28679 .

Avec l'aimable autorisation de la famille Dib.

() Bibliothèque nationale de France

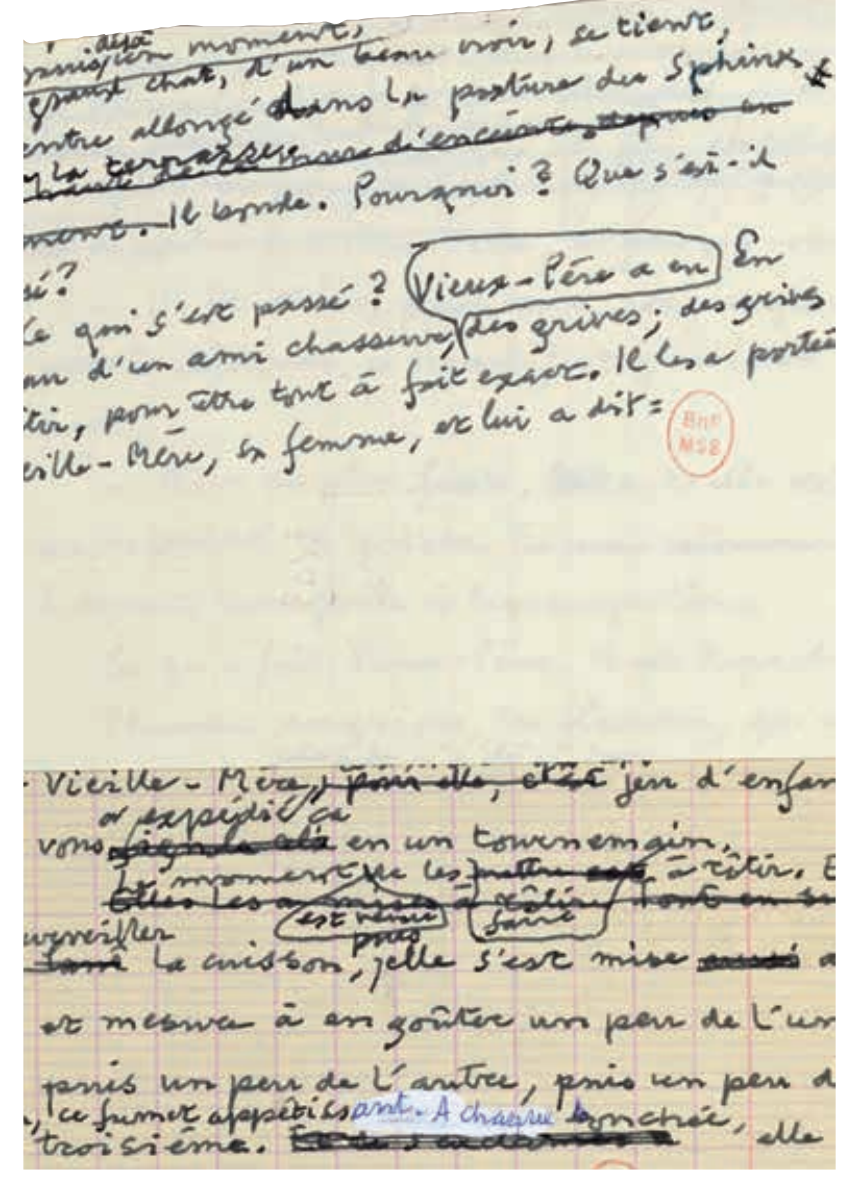

Fig. $5 \mathrm{~b}:$ Incipit mis au net de la troisième version - Collage sur fol. 6.

Boîte 38 bis, cote Naf 28679.

Avec l'aimable autorisation de la famille Dib.

(c) Bibliothèque nationale de France 


\section{L'Hippopotame qui se trouvait vilain}

\section{Les avant-textes}

Composés de quarante-quatre feuillets dactylographiés numérotés au format A4, les avant-textes de L'Hippopotame qui se trouvait vilain, plus nets que ceux de L'Histoire du chat qui boude, sont constitués de trois versions tapuscrites, dont deux similaires (ci-après tapuscrits 1 à 4), avec quelques annotations manuscrites, de ce que l'auteur dans ses brouillons, comme l'éditeur en quatrième de couverture, présente comme «un conte original de pure imagination».

Le tapuscrit 1, intitulé Le bébé hyppopotame [sic], occupe les feuillets 1 à 5 . Il est introduit par une présentation à la première personne, sorte de monologue intérieur révélateur de la manière dont le récit pour enfants vient à son auteur, via l'oralité, les récits faits en famille. Cette oralité disparaîtra des versions ultérieures et de la publication, sans que l'on puisse expliquer pourquoi.

Voilà... C'est une histoire que je n'ai pas écrite encore. Je ne sais pas si je l'écrirai un jour. Je l'ai racontée une fois seulement à ma petite fille. Elle est là... si elle veut venir aussi. Chaque fois que je la raconte, je pense la raconter d'une manière différente... Alors voilà...

Il était une fois... 16

On reconnaît dans la formule : «Il était une fois...» l'introït du conte traditionnel - et c'est comme «conte» que Mohammed Dib présente ce récit dans son premier brouillon. Formule et référence au conte disparaîtront des versions ultérieures, mais réapparaîtront, comme nous l'avons noté, en quatrième de couverture de l'ouvrage publié.

Le tapuscrit 1 donne ensuite au récit sa forme globalecelle d'un conte de randonnée. Mais la fin de cette version est surprenante, comme si le conteur souhaitait se débarrasser brusquement d'un récit dont les enchaînements le fatiguent (fig. 7a).

Plus développé, le tapuscrit 2, intitulé Un drôle de bébé hyppopotame [resic], est composé de treize feuillets numérotés, dont certains dactylographiés au verso de documents journalistiques de la décennie 199017. L'introduction disparaît; la forme globale du récit est maintenue, ainsi qu'au fil de la narration quelques indices d'un dialogue entre le conteur et son auditeur (ex. : «Ces bêtes qu'on ne rencontre qu'en Afrique, tu ne sais peut-être pas à quoi

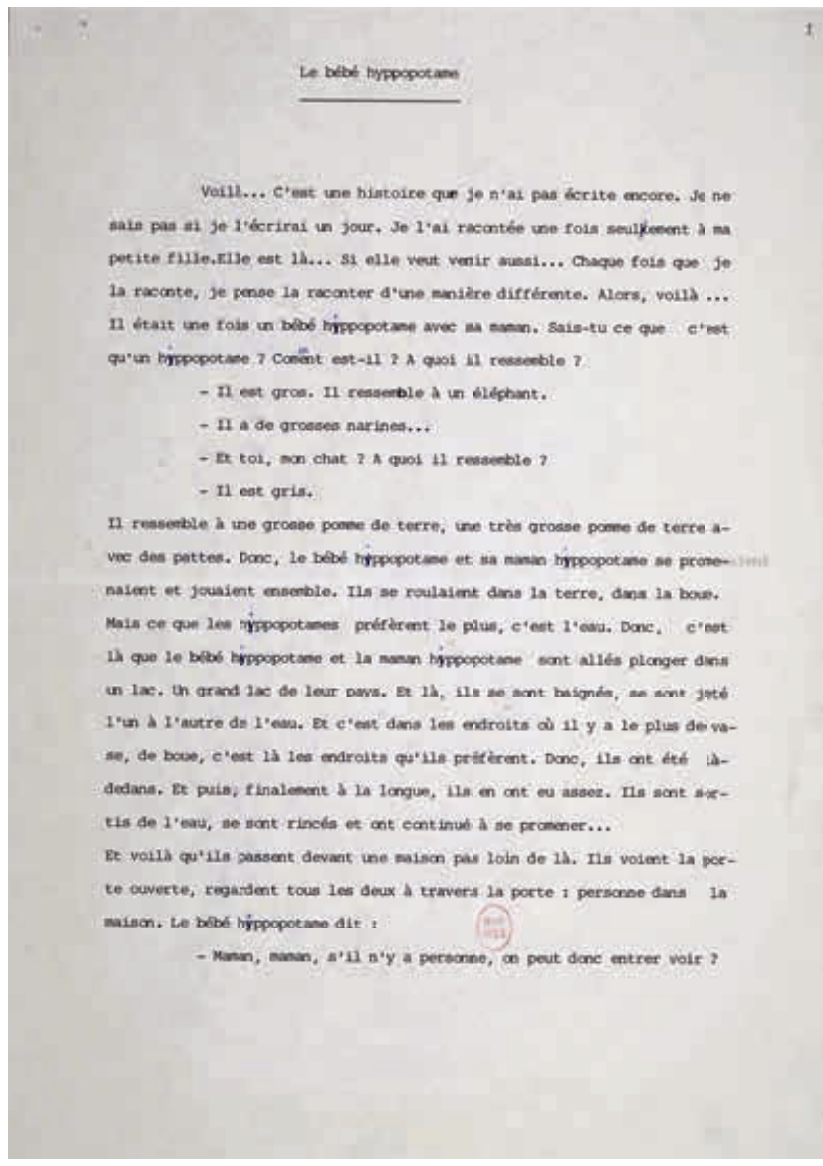

Fig. 6 : Page de titre du premier tapuscrit.

Boîte 38bis, cote Naf 28679. Avec l'aimable autorisation de la famille Dib. (C) Bibliothèque nationale de France

elles ressemblent... Tu sauras aussi que le plaisir...», p. 1, je souligne); un dénouement plus satisfaisant apparaît, qui sera celui du récit publié.

Les deux dernières versions (les tapuscrits 3 et 4 , également de treize pages chacun) reprennent, à quelques corrections près, le même texte sous un titre corrigé, Un drôle de bébé hyppopotame. Dans toutes les versions dactylographiées, le récit reste raconté au passé.

À cet ensemble narratif, l'œuvre publiée - d'un format très réduit $(14,5 \times 17,5)$ et bénéficiant d'un nouveau titre, plus précis : L'Hippopotame qui se trouvait vilain - n'a plus qu'à actualiser le récit (passage des temps du passé au présent), à y ajouter un peu de couleur locale, à l'aide

\footnotetext{
16. Tapuscrit 1, fig. 6 .
}

17. Dossier sur les prix 1992 à 1994 de la Fondation Noureddine Aba et programme d'une rencontre du Parlement international des écrivains sur les «littératures déplacées» (novembre 1994), cela ne permettant pas pour autant de les dater avec exactitude. 


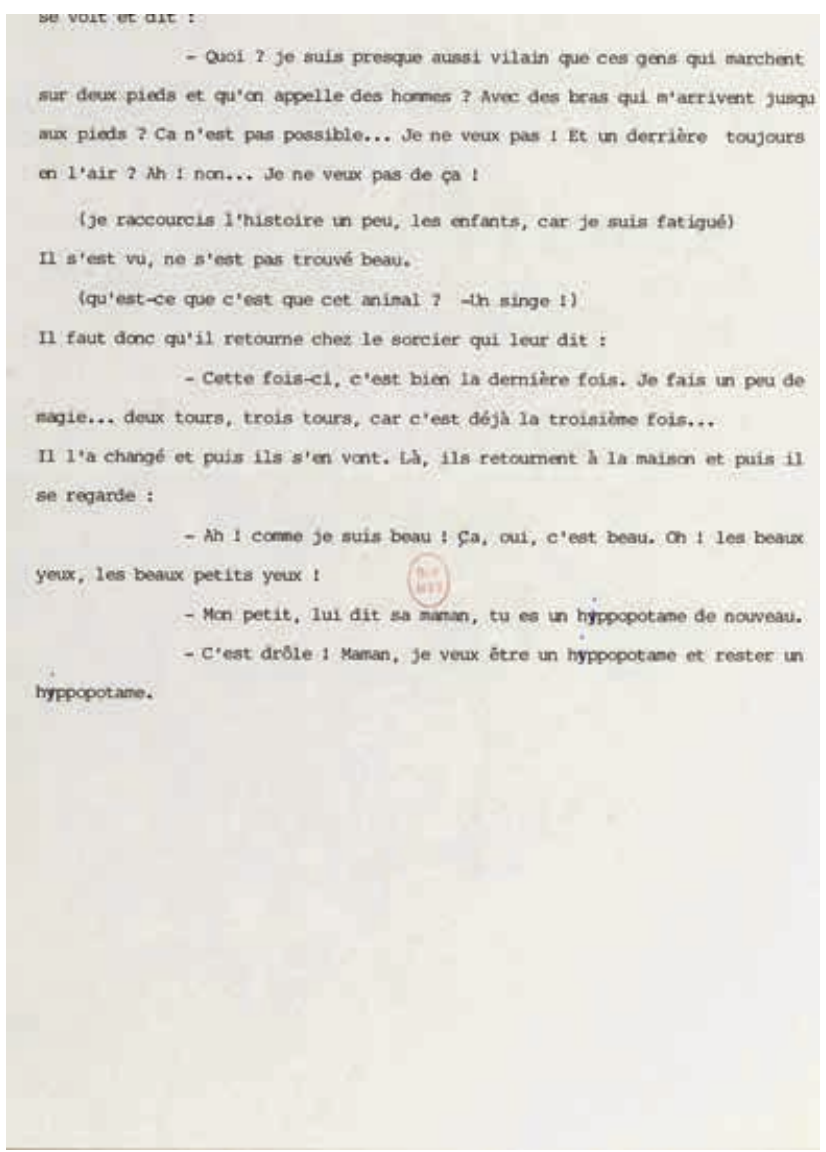

Fig. 7a : Page de fin, tapuscrit 1. Boîte 38bis, cote Naf 28679. Avec l'aimable autorisation de la famille Dib. (C) Bibliothèque nationale de France

d'un vocabulaire connotant plus l'Afrique équatoriale 18 ou en imaginant tout un jeu d'incantations pour le sorcier ensemble de procédés qui ont aussi pour effet de renforcer

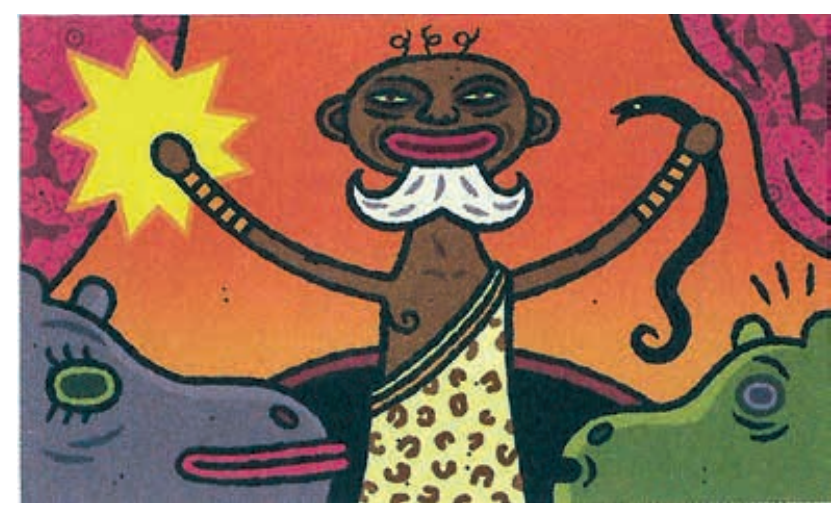

Fig. 8. Vignette illustrant L'Hippopotame qui se trouvait vilain. Emmanuel Kerner, illustrateur, Paris, Albin Michel Jeunesse, 2001. Collection particulière de l'auteur.

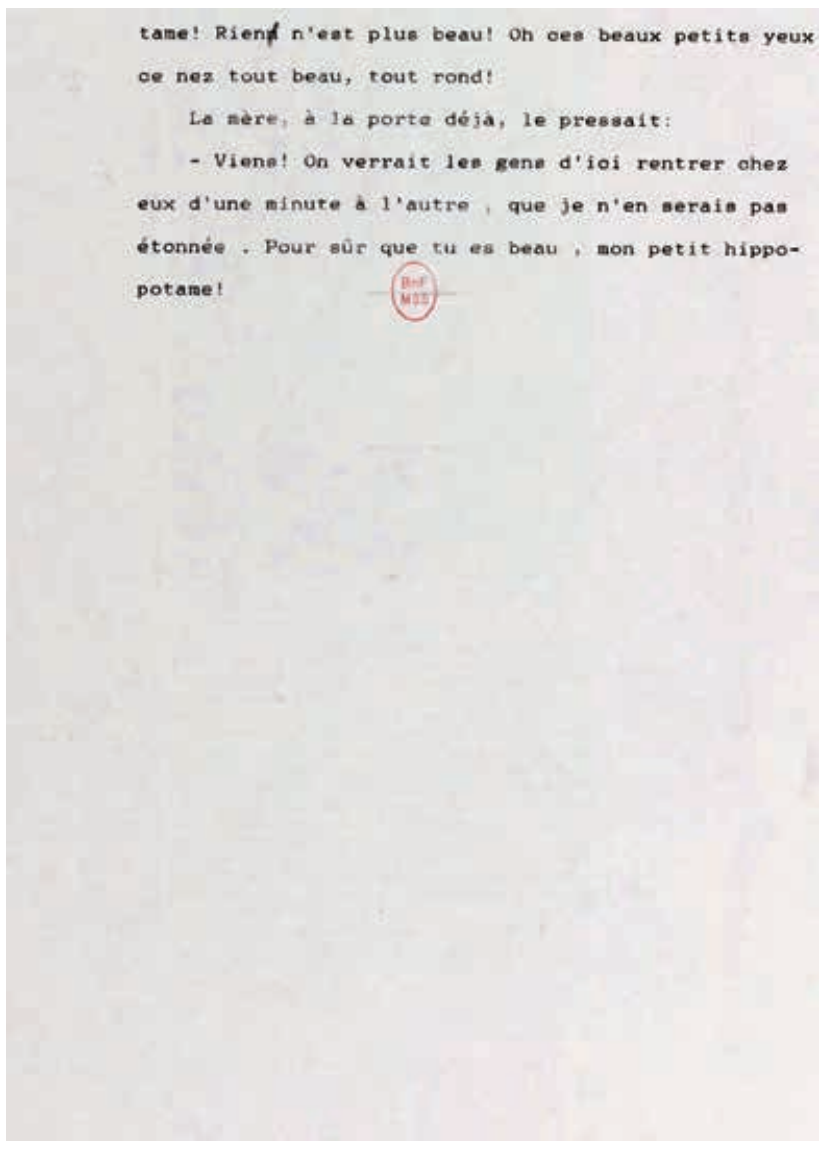

Fig. 7b. Page de fin, tapucrits 3 et 4. Boîte 38bis, cote Naf 28679.

Avec l'aimable autorisation de la famille Dib. (c) Bibliothèque nationale de France

sa dimension contique. Le texte se clôt sur la formule traditionnelle : «Nous sommes allés... pour toi...», présente dans tous les récits pour enfants de Dib.

\section{L'illustration}

Emmanuel Kerner a choisi d'illustrer ce texte de petites vignettes très naïves, le plus souvent rectangulaires, placées au-dessus ou en dessous d'un texte plus abondant que dans les ouvrages précédents, au service duquel elles figurent avec discrétion (pas plus d'une par double page).

18. Par exemple, le «grand lac de leur pays» (dans les tapuscrits 1 à 4 ) devient un «marigot de leur pays» et le «grand magicien» disparaît au profit du «sorcier $» .$. 


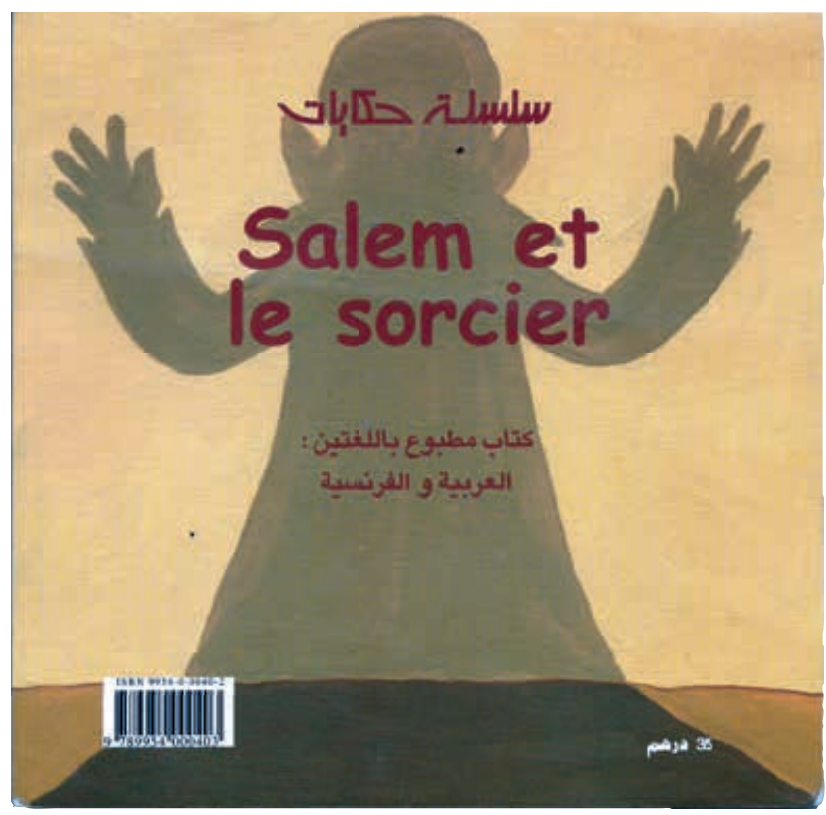

Fig. 9. Couverture format carré $(19$ x $19 \mathrm{~cm})$ de Salem et le sorcier. Seconde édition : Casablanca, Yomad, 2003.

Collection personnelle de l'auteur.

\section{Salem et le sorcier}

Salem et le sorcier est l'un des tout derniers écrits de Mohammed Dib, publié en 2000 par une maison d'édition marocaine, puis réédité en version bilingue française et arabe 19 juste après la mort de l'auteur, en 2003 - les deux ouvrages dans un format calendrier carré $(19 \times 19 \mathrm{~cm})$, l'un des côtés de la couverture portant le titre en arabe, l'autre en français. Comme on ne dispose d'aucun brouillon, on ignore si le texte en arabe est autographe.

\section{Le récit}

Il s'agit, cette fois encore, d'un conte de randonnée : Salem est employé par «un méchant sorcier» qui le tyrannise et l'épuise au travail. Lorsque le sorcier lui demande d'aller ouvrir la porte, le petit garçon refuse de se lever. Furieux, le sorcier ordonne à son chien d'aller mordre l'enfant; le chien refuse à son tour. Le sorcier sollicite alors son bâton; mais le bâton refuse de bastonner. Le sorcier jette le bâton dans le feu, qui refuse de s'allumer... et ainsi de suite jusqu'à récapitulation et conclusion : «Le sorcier dut aller ouvrir lui-même. Depuis ce jour, Salem vit libre et heureux.»

Texte et illustration dénotent un ancrage résolument arabo-musulman : vêtements et habitat y renvoient clairement, et le méchant sorcier interpelle «par Iblis»!

Mais ce récit nous intéresse surtout du fait d'une découverte : six ans avant sa publication chez Yomad,

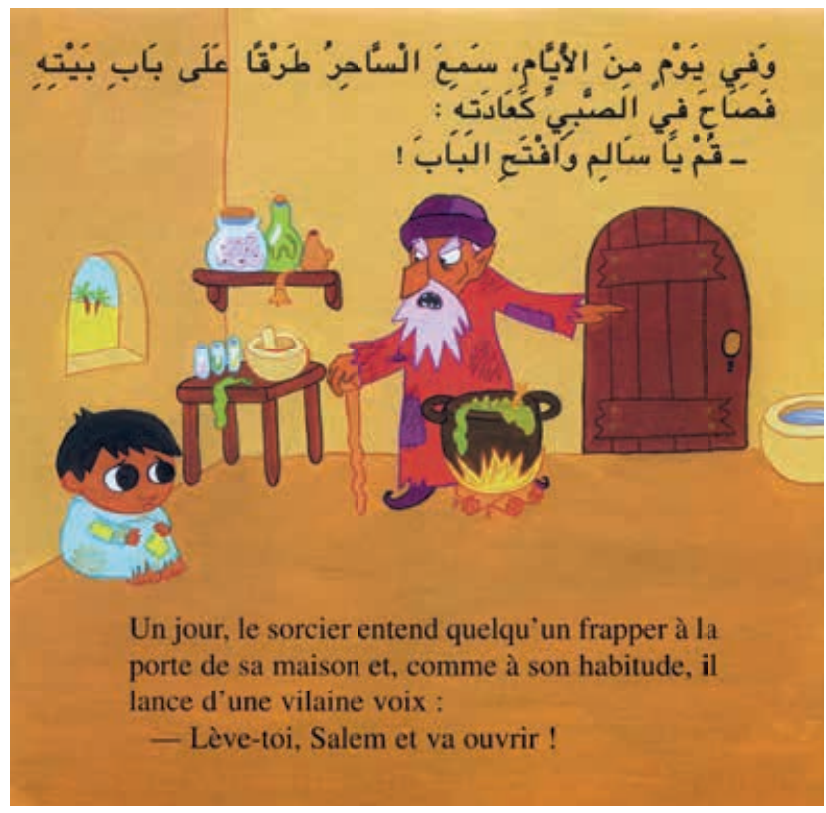

Fig. 10. Ancrage du récit dans un contexte arabo-musulman. Seconde édition : Casablanca, Yomad, 2003.

Collection personnelle de l'auteur.

cette histoire se trouvait intégralement insérée dans le roman L'Infante maure ${ }^{20}$, au sein d'un contexte énonciatif particulièrement significatif : en effet, ce roman met en scène une enfant, Lylli Belle, née d'un couple mixte (père maghrébin, mère nord-européenne) tentant de concilier les univers complémentaires et opposés qu'elle porte en elle.

Il est intéressant de noter que ce conte né de l'imaginaire paternel et censé représenter l'apport maghrébin dans la culture de Lylli apparaît ici finalement proche de l'imaginaire occidental - pour preuve une chanson populaire comme «Biquette», récit de randonnée comportant un enchaînement du même type, qu'on retrouve dans toute l'Europe. Et c'est ici l'enfant qui, en renversant les codes habituels selon lesquels ce seraient d'ordinaire les aînés qui racontent aux plus jeunes, narre à sa mère «une histoire que papa a rapportée de là-bas, de son pays ».

Dans ce roman, la petite fille clôt son récit sur la formule habituelle :

Nous sommes allés tout au long du chemin et nous avons trouvé un sac de perles : les grosses pour moi, les petites pour toi... 21

19. Sur les enjeux économiques de l'édition bilingue du roman marocain pour enfants, voir le site Takam Tikou < http://takamtikou.bnf.fr/ >

20. Mohammed Dib, L'Infante maure, Paris, Albin Michel, 1994.

21. Ibid., p. 125. 
Après la lecture, la mère, davantage surprise par la clausule que par la forme de l'histoire elle-même, commente :

- Quelle jolie histoire! Et qui finit bien.

- N'est-ce pas, maman? Je te l'ai dit.

- Je n'ai seulement pas compris pourquoi on trouve ces perles sur la route, si elles ont le moins du monde quelque chose à voir avec l'histoire, mais c'est joli aussi22.

Et la petite fille de conclure :

Là-bas, les contes finissent toujours comme ça, avec des perles 23 .

On peut donc formuler l'hypothèse que l'édition du récit fait par le père à sa fille, puis par celle-ci à sa mère, résulte d'un simple copier-coller après autorisation de l'auteur, expliquant ainsi l'absence de tout brouillon et l'omission de la formule canonique dont nous avons noté la présence dans tous les autres recueils pour enfants de Dib - celle-ci participant dans le roman, du fait de la situation d'énonciation particulière, d'une poétique de réconciliation des cultures qui disparaît forcément dans la publication autonome 24 .

\section{L'illustration}

Nous avons pu interroger Virginie Soumagnac, illustratrice de ce dernier récit, qui nous a fourni quelques indications sur son travail25:

- Elle ne connaissait pas l'auteur et n'a eu aucun contact avec lui préalablement ou pendant son travail.

- Elle n'a jamais eu connaissance des avant-textes.

- Sollicitée directement par la maison d'édition, elle a reçu un texte en français par voie électronique, et ce n'est qu'à la publication qu'elle a découvert que l'édition était bilingue : elle avait seulement été avertie, sans autre explication, qu'elle devrait laisser un double espace pour le texte, en haut et bas de page.

- La consigne donnée par l'éditeur était de dessiner «un univers de personnes très pauvres (maison, vêtements...) et un sorcier le plus terrifiant possible».

L'illustratrice souligne qu'elle a fidèlement respecté ces consignes - la seule liberté qu'elle ait prise tient au dernier dessin qui montre Salem partant «avec tous les insoumis», ce que ne spécifie pas le texte.

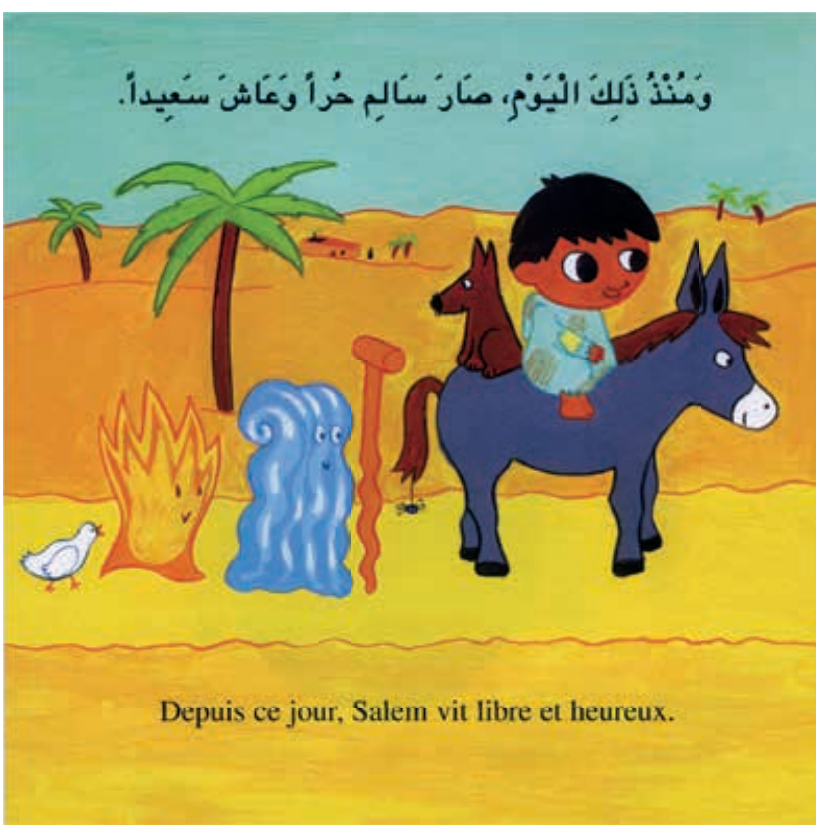

Fig. 11. Dénouement de Salem et le sorcier. Collection personnelle de l'auteur.

\section{Conclusion}

Génétique textuelle, génétique éditoriale, collaboration auteur/illustrateur... de cette passionnante recherche, nous pouvons tirer plusieurs conclusions intéressant le généticien :

- Si l'idée de recueillir des récits de la tradition orale maghrébine vient assez tôt dans l'œuvre pour enfants de Dib (voir les divers récits de Baba Fekrane), le travail d'écriture

\section{Ibid., p. 125.}

23. Ibid., p. 125

24. Je remercie vivement Rudolf Mahrer, qui a attiré mon attention sur l'existence de ce même procédé dans la tradition populaire occidentale et de la parenté de ce conte avec une chanson comme «Biquette» recueillie par Jean-Baptiste Weckerlin, Chansons et Rondes enfantines, Garnier, 1870. Les conséquences que l'on peut en tirer en termes d'analyse poétique du conte mais surtout du roman sont multiples et fort intéressantes; elles nous conduiraient néanmoins bien au-delà du but visé par cet article.

25. Dans un échange de mails que nous avons eu préalablement à la rencontre sur les brouillons d'écrivains. 
ou de réécriture spécifique au genre est plus tardif, comme le montrent les brouillons manuscrits ou tapuscrits. Nous formulons l'hypothèse qu'il est contemporain de la période où Dib, lui-même grand-père, eut à raconter des histoires de ce type à ses petits-enfants (décennie 1985-1995) et qu'il devient à ce moment-là un procédé de la poétique dibienne en général. Ce que vient confirmer la mise en abyme que l'on trouve dans L'Hippopotame qui se trouvait vilain ou de manière inversée dans L'Infante maure, où c'est l'enfant qui raconte à l'adulte.

- Les récits pour enfants de Mohammed Dib accompagnent l'ensemble de son œuvre dans un double mouvement de valorisation de valeurs humanistes universelles (justice, liberté individuelle, solidarité entre «subalternes »...) et de retour à un ancrage explicitement arabo-musulman. Que ce double mouvement se fasse de manière très équilibrée 26 à travers ce genre (ou plus explicitement encore dans L'Infante maure sous forme d'un microrécit raconté par l'enfant) est significatif d'une volonté de renversement des codes d'une part et d'une volonté d'harmoniser des imaginaires culturels trop souvent considérés comme antagonistes d'autre part.

L'existence des brouillons tapuscrits de L'Hippopotame qui se trouvait vilain et manuscrits du Chat qui boude dans les archives Dib de la BnF permet en outre diverses analyses d'ordre génétique :

- Les brouillons les plus fournis et les plus intéressants sont indéniablement ceux du Chat qui boude, parce qu'ils sont abondamment annotés, et qu'ils présentent un exemple, rare dans ce genre, de réécriture d'un même texte avec vingt ans d'écart.

- En mettant l'accent sur le passage d'une poétique du récit oral au récit écrit qui interdit toute variation du récit («Chaque fois que je la raconte, je pense la raconter d'une manière différente "), l'incipit de L'Hippopotame qui se trouvait vilain indique le récit oral comme origine de ce type d'écriture, chez Dib comme chez la plupart des écrivains-conteurs, les différences entre les brouillons et leur conclusion précipitée faisant l'aveu d'une frustration de l'auteur face au conteur. La «posture» du conteur face à son public engage une relation pédagogique que l'on retrouve aussi dans certains romans ou nouvelles de la première période d'écriture de Dib - on pense à la relation entre Comandar et Omar dans la trilogie Algérie, au sujet de laquelle on a pu parler d'une volonté de «pédagogie militante » dont l'écrivain «se défera vite» dans son œuvre ultérieure 27 , en même temps qu'il se libérera du point de vue réaliste.

- Une étude plus systématique des couvertures et de l'illustration dans ces divers textes et leurs rééditions apporterait d'autres éléments intéressant l'élaboration de ces œuvres hybrides. Mais cela passe par davantage de contacts avec les illustrateurs que nous avons pu en avoir.

26. Sauf peut-être dans Salem et le sorcier, texte ultime, où apparaît, à travers l'illustration, mais aussi parfois à travers le texte, un côté exotique si peu dibien à notre avis.

Après rédaction de cet article, nous avons trouvé en Algérie une nouvelle édition en arabe de Salem et le sorcier, qui reprend très exactement, dans un format carré $21 \times 21$, le texte et les illustrations de l'édition marocaine : Alger, éd. Sédia, 2007.

27. Voir Charles Bonn, Lecture présente de Mohammed Dib, Alger, ENAL, 1988, chap. «La tension didactique de L'Incendie ». 
GUY DUGas est professeur émérite de Littérature générale et comparée à l'université Montpellier III. Il est l'auteur d'une thèse pionnière sur La Littérature judéo-maghrébine d'expression française et d'anthologies sur les francophonies maghrébines aux éditions Omnibus (Algérie. Un rêve de fraternité; Tunisie. Rêve de partages; Maroc. Les villes impériales...). Chercheur associé à l'ITEM, équipe «Manuscrits francophones», il a pris en charge l'édition génétique de l'œuvre d'Albert Memmi chez CNRS Éditions et travaille actuellement sur son journal intime, après avoir dirigé l'édition des Portraits (CNRS Éditions, coll. «Planète libre», 2015). Dernier ouvrage publié : Journal de guerre (1939-1943) d'Albert Memmi, CNRS éditions, coll. «Biblis», 2019.

dugas.montp3@gmail.com

\section{Genèse du récit maghrébin pour enfants. Le cas de Mohammed Dib}

Grand Prix de la Francophonie en 1994, Mohammed Dib (1920-2003), l'un des écrivains maghrébins les plus reconnus, est l'auteur de quatre récits pour enfants dont les avant-textes sont partiellement déposés à la Bibliothèque nationale de France. À partir de ce matériau complété d'entretiens avec des illustrateurs et éditeurs, cet article analyse la genèse de ces récits, en s'attachant plus particulièrement à l'évolution de la relation texte-image au fil des trois éditions de L'Histoire du chat qui boude, des tapuscrits de L'Hippopotame qui se trouvait vilain et du texte bilingue (français/arabe) de Salem et le sorcier pour lequel on ne dispose d'aucun brouillon. Un tel travail sur les récits pour enfants révèle des filiations, divers emprunts aux traditions contiques maghrébine et occidentale, ainsi que certains intertextes internes à l'œuvre pour adultes de Mohammed Dib.

Winner of the Grand Prix de la Francophonie in 1994, Mohammed Dib (1920-2003), one of the best-known writers of the Maghreb, is the author of four stories for children. The preparatory material is held by the National Library of France. Based on this material, complemented by conversations with illustrators and publishers, this article studies the genesis of these stories, focusing in particular on the evolution of the relationship between text and image through the three editions of L'Histoire du chat qui boude, several typescripts of L'Hippopotame qui se trouvait vilain and through the bilingual text (arabic-french) of Salem et le sorcier, which has no draft. This type of work on narratives for children reveals a number of influences, a substantial borrowing from the Maghreban and Western traditions of tales, and an intertextual relation with some aspects of Mohammed Dib's work for adults.

Als Gewinner des Grand Prix de la Francophonie des Jahres 1994 ist Mohammed Dib (1920-2003) einer der bekanntesten Schriftsteller aus dem Maghreb. Er ist der Autor von vier KinderErzählungen, deren avant-textes in der französischen Nationalbibliothek liegen. Ausgehend von diesem Material, welches durch Gespräche mit Illustratoren und Herausgebern ergänzt wird, analysiert der vorliegende Artikel die Genese dieser Erzählungen, und zwar mit einem Fokus auf die Beziehung zwischen Texten und Bildern am Leitfaden der drei Ausgaben von L'Histoire du chat qui boude, zahlreicher Typoskripte von L'Hippopotame qui se trouvait vilain und schließlich des zweisprachigen (arabisch-französischen) Texts von Salem et le sorcier, zu dem sich kein Entwurf erhalten hat. Eine solche Studie über die Kinder-Erzählungen zeigt Verwandtschaften, Einflüsse maghrebinischer und westeuropäischer
Erzähl-Traditionen, wie auch interne Verflechtungen mit Mohammed Dibs Literatur für Erwachsene auf.

Premiado por la Francofonía en 1994, Mohammed Dib (19202003), uno de los escritores magrebís más reconocidos, es el autor de cuatro relatos para niños, cuyos pre-textos están depositados en la Biblioteca Nacional de Francia. A partir de ese material, completado con entrevistas a ilustradores y editores, este artículo analiza la génesis de esos relatos, centrándose más particularmente en la evolución de la relación texto-imagen a lo largo de las tres ediciones de L'Histoire du chat qui boude, de los dactiloscritos de L'Hippopotame qui se trouvait vilain y del texto bilingüe (francés/ árabe) de Salem et le sorcier, del cual no se dispone de ningún borrador. Un trabajo de este tipo sobre los relatos para niños pone de manifiesto filiaciones, elementos de la tradición cuentística magrebí y occidental, así como algunos intertextos internos de la obra para adultos de Mohammed Dib.

Grande prémio da Francofonia em 1994, Mohammed Dib (19202003), um dos mais conhecidos escritores do Magrebe, é autor de quatro histórias para crianças, cujos antetextos se acham depositados na BnF. A partir desse material, complementado por entrevistas com ilustradores e editores, é analisada a génese das histórias, com enfoque na evolução da relação texto-imagem das três edições de L'Histoire du chat qui boude, dos dactiloscritos de L'Hippopotame qui se trouvait vilain, e do texto bilingue (francês/ árabe) de Salem et le sorcier, do qual não existe qualquer rascunho. Tal trabalho revela certas filiações, legados das tradições narrativas magrebinas e ocidentais, bem como algumas ligações internas às obras para adultos de Mohammed Dib.

Grand Prix della Francofonia nel 1994, Mohammed Dib (19202003), uno degli scrittori magrebini più noti, è l'autore di quattro racconti per l'infanzia i cui avantesti sono conservati alla BnF. A partire da questo materiale, integrato da interviste a illustratori ed editori, l'articolo analizza la genesi di questi racconti, soffermandosi in particolare sull'evoluzione della relazione tra testo e immagine nelle tre edizioni di L'Histoire du chat qui boude, dei dattiloscritti di L'Hippopotame qui se trouvait vilain e del testo bilingue (francese/arabo) di Salem et le sorcier, del quale non possediamo nessuno "scartafaccio". Questo lavoro sui racconti per l'infanzia rivela dei prestiti dalle tradizioni orali magrebine e occidentali ma anche alcune intertestualità interne alle sue opere per adulti. 